\title{
Characterization of the human endogenous retrovirus K Gag protein: identification of protease cleavage sites
}

\author{
Benjamin Kraus, Klaus Boller, Andreas Reuter and Barbara S Schnierle*
}

\begin{abstract}
Background: Viral genomes of the human endogenous retrovirus K (HERV-K) family are integrated into the human chromosome and are transmitted vertically as Mendelian genes. Although viral particles are released by some transformed cells, they have never been shown to be infectious. In general, gammaretroviruses are produced as immature viral particles by accumulation of the Gag polyproteins at the plasma membrane, which subsequently bud from the cell surface. After release from the cell, Gag is further processed by proteolytic cleavage by the viral protease (PR), which results in morphologically mature particles with condensed cores. The HERV-K Gag polyprotein processing and function has not yet been precisely determined.

Results: We generated a recombinant poxvirus, encoding the human endogenous retrovirus $\mathrm{K}$ consensus gag-propol genes (MVA-HERV- $\mathrm{K}_{\text {con }}$ ) and obtained high levels of HERV-K Gag expression. The resulting retroviral particle assembled at the plasma membrane, as is typical for gammaretroviruses; and immature as well as mature retrovirus-like particles (VLPS) were observed around the infected cells. VLPs were purified, concentrated and separated by two-dimensional gel electrophoresis. The HERV-K Gag fragments were identified by mass spectroscopy and N-terminal sequencing which revealed that HERV-K Gag is processed into MA, a short spacer peptide, p15, CA and NC.

Conclusion: The cleavage sites of HERV-K Gag were mapped and found to be highly conserved among HERV-K genomes. The consensus HERV-K gag gene used in this study is known to support viral, infectivity [1], and thus the cleavage sites that were mapped in this study for all the Gag components are relevant for HERV-K infectivity.
\end{abstract}

\section{Background}

Human endogenous retroviruses (HERVs) are relics of evolutionary ancient viral infection events which involved insertion into the germ line and are now transmitted vertically. These retroviral genomes are chromosomally integrated in all nucleated cells of an individual and their sequences, including solitary LTRs, comprise about $8 \%$ of the human genome. HERVs are classified by the single letter amino acid code for the tRNA specific to the primer-binding site (PBS) used to initiate reverse transcription. At present, 11 distantly related HERV groups with a tRNA lysine (K) PBS exist (reviewed in [2]). One of these, the HERV-K/HML-2 (hom) group is the only known endogenous retrovirus

\footnotetext{
* Correspondence: schba@pei.de

Paul-Ehrlich-Institut, Paul-Ehrlich-Strasse 51-59, 63225 Langen, Germany
}

group encoding all structural and enzymatic proteins (proteins encoding the viral core [Gag], UTPase/protease [PR], polymerase [Pol], RNaseH, integrase [Int]), and envelope [Env]) and the accessory protein Rec with functional similarity to the HIV Rev protein [3]. In general, HERV-K gene expression is repressed in somatic cells; however, reactivation of HERV-K proviruses coding for all viral proteins has been described for human teratocarcinomas [4], as well as for melanomas [5], and ovarian cancer [6], [7]. Although full-length genomic mRNA and viral particles are detectable, HERV-K has never been shown to be infectious and the viral genome is only known to be transmitted via the germ line. However, the HERV-K family may have replicated less than 1 million years ago and a consensus HERV-K sequence has been constructed that likely resembles the progenitor of HERV-K that entered the human genome within

\section{() Biomed Central}


the last few million years. Infectious particles could be generated from this consensus HERV-K provirus sequence [1], [8]. This raises concerns that recombination between different HERV-K genomes might be able to form infectious viral genomes able to reinfect human cells, especially germ cells.

Retroviral Gag polyproteins play a central role in viral particle formation and are responsible for particle assembly, release and infectivity. Gag itself is sufficient for the formation of virus-like particles (VLPs). It is first synthesized as a polyprotein and is further processed in viral particles by the viral protease (PR) to produce the major structural proteins MA (matrix), CA (capsid) and $\mathrm{NC}$ (nucleocapsid). This genetic organization as a polyprotein precursor that is subsequently cleaved exploits the protein-protein interactions needed for virion assembly to ensure the incorporation of the enzymes needed for replication, maturation and infection. Mutation of the retroviral PR prevents cleavage but does not prevent assembly of Gag at the plasma membrane and virus release; however, the resulting particles remain immature, with a changed morphology that never shows collapsed cores, and are not infectious.

Cell associated HERV-K particles budding from human teratocarcinoma cell lines have been shown to exist exclusively as morphologically immature particles [9]. However, a functional protease has been detected [10]. This raises the possibility that a defect in cleavage is caused by mutated cleavage sites. Therefore, we have characterized here the HERV-K Gag protein processing based on the consensus HERV-K genome sequence [1].

\section{Results}

HERV-K Gag processing is mediated by the viral protease

Processing of retroviral Gag polyproteins in immature viral particles is mediated by the viral protease $(\mathrm{PR})$ and induces a reorganization of the internal virion structure, termed maturation, which becomes morphologically visible as collapse of the viral core. Mutation or deletion of PR prevents cleavage but does not prevent assembly, resulting in immature particles. A functional protease has been described for HERV-K [10]. To analyze a potential defect in Gag cleavage and to obtain high amounts of HERV-K particles, HERV-K Gag expression with the help of the modified vaccinia virus Ankara (MVA) was employed. The consensus HERV-K Gag-PRPol gene [1] was cloned into the MVA expression vector pIII-mH5 [11] generating the plasmid pIII-HERV-K $\mathrm{K}_{\text {con }}$, where expression is controlled by a strong early/late promoter. In addition, we constructed a protease-deficient variant of that construct, named pIII-HERV-K $\mathrm{K}_{\text {con- }}$ pro. The transfer plasmids pIII-HERV-K $\mathrm{K}_{\text {con }}$ and pIIIHERV-K conpro $^{-}$were transfected into HEK $293 \mathrm{~T}$ cells, and these were infected with MVA to induce transient expression of the HERV-K gag/pol genes controlled by the vaccinia virus specific promoter. Subsequent Western blot analysis of cell lysates (lys) and concentrated supernatants (sup) revealed that HERV-K Gag/Pol was expressed after MVA infection (Figure 1A, lanes 2, 3, 5 and 6) and particles could be pelleted by ultracentrifugation from supernatants of these cells (lanes 3 and 6). Particles derived from pIII-HERV-K $\mathrm{K}_{\text {con }}$ contained mainly processed Gag, since the Gag precursor could only be detected in cell lysates and not in the supernatants (Figure 1A, lanes 2 and 3). However, only the Gag precursor could be detected in cell lysates and viral particles from pIII-HERV-K con pro $^{-}$-transfected cells (Figure $1 \mathrm{~A}$, lanes 5 and 6). Consequently, processing of HERV$\mathrm{K}$ Gag was dependent on the presence of the functional retroviral protease and MVA infection was not responsible for cleavage products.

\section{HERV-K particles are released from cells infected with recombinant HERV-K Gag/Pol-expressing MVA}

After showing the value of this expression system for HERV-K Gag processing analysis, we generated a recombinant MVA stably expressing the HERV-K gag/ pol gene (MVA-HERV- $K_{\text {con }}$ ). The recombinant virus was constructed as described previously, using K1L selection [11]. Quality control by PCR and Western blot analysis confirmed the proper expression of HERV-K Gag/Pol in cells infected with MVA-HERV-K $\mathrm{K}_{\text {con }}$ (Figure 1B, lanes 4 and 5). To further demonstrate that MVA does not alter the cleavage pattern of Gag, we additionally analyzed cell lysates and concentrated viral particles from HEK 293T cells transfected with the HERV-K Gag-PR-Pol expression plasmid which expresses HERVK Gag/Pol driven by a CMV promoter $48 \mathrm{hrs}$ after transfection [1]. Fragments, representing Gag and capsid or partial cleavage products thereof had the same size independently if HERV-K Gag/pol was expressed in the presence or absence of MVA (Figure 1B, lanes 3 and 5). This is further evidence that processing of HERV-K Gag mediated by the retroviral protease. In addition, MVAdriven protein expression is more efficient than plasmid-based expression, since only one-third of the MVAinfected cell material was loaded in Figure 1B and the infected cells were already harvested $24 \mathrm{hrs}$ after infection.

To determine if virus-like particles are formed by

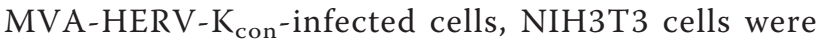
infected at an MOI of 5 and the cells were fixed $24 \mathrm{~h}$ after infection and analyzed by electron microscopy. Retroviral particles were detected budding at the cell surface (Figure 2A) and Figure 2B shows free immature retroviral particles. Figure $2 \mathrm{C}$ shows the presence of incoming poxviral particles as typically seen after MVA infection, as well as mature retroviral particles with 


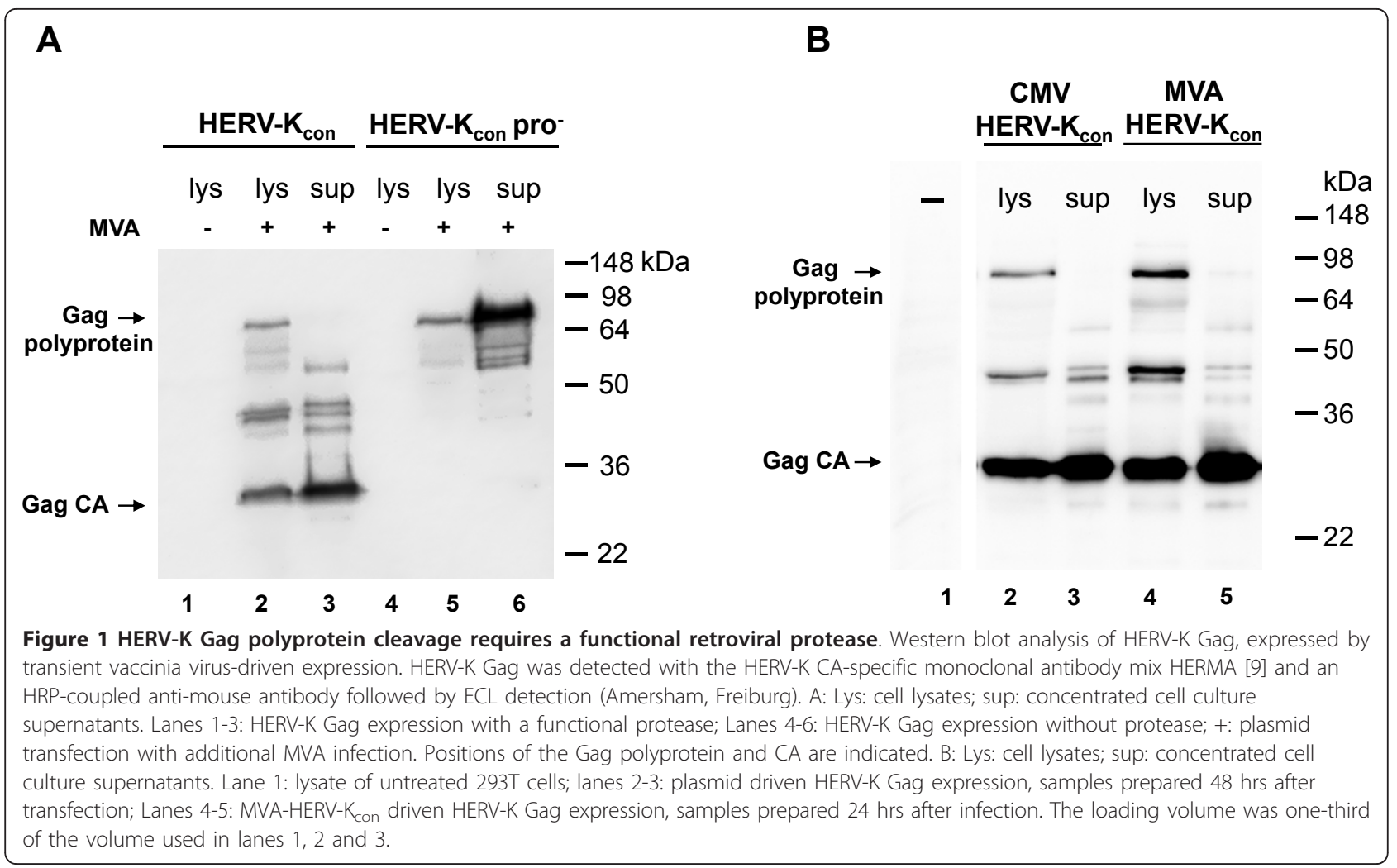

condensed cores (Figure 2C). Therefore, supernatants of MVA-HERV-K $\mathrm{K}_{\text {con }}$-infected cells could be used to generate large amounts of HERV-K virus-like particles.

\section{Separation of processed Gag fragments isolated from VLPs and N-terminal sequence determination}

The HERV-K Gag protein has a functional organization typical for retroviruses. In silico predictions imply an Nterminal matrix protein (MA) with an $\mathrm{N}$-terminally myristoylated head, which usually directs Gag to cell membranes. At the $\mathrm{C}$-terminus are two Zink-finger motifs, suggesting the location of the nucleocapsid (NC) protein. The NC binds RNA and enables the packaging of the viral genome. The central region of the HERV-K Gag has homology to retroviral capsid (CA) proteins. CA usually homo-oligomerizes and determines the particle morphology. Retroviruses also have other proteins which are generally designated by their molecular weight, such as p12 of murine leukemia virus; however, their function is still unknown [12]. Cleavage of in vitrotranslated HERV-K Gag by its purified protease expressed in E. coli has been shown before [13], but the exact boundaries and the precise size of processed HERV-K Gag proteins were unknown.

To study HERV-K Gag processing, HEK 293T cells were infected at an MOI of 5 for $24 \mathrm{~h}$ with MVA- 
HERV-K $\mathrm{K}_{\text {con }}$ and cell supernatants were collected and concentrated by ultracentrifugation through a sucrose cushion. To analyze the Gag cleavage products in VLPs, two-dimensional (2-D) electrophoresis was employed. The concentrated virus-like particles were first subjected to isoelectric focusing, followed by SDS-PAGE as the second dimension (Figure 3). The gel was stained with Coomassie dye and single proteins were cut out and analyzed by mass spectrometry (MS). With the exception of one spot (Figure 3A, indicated by a black circle), all spots analyzed corresponded to HERV-K sequences. As a control, the same procedure was performed with wt MVA-infected cells and, as expected, no proteins were detected in the 2-D analysis (data not shown). Figure 3B summarizes the results, and color coding indicates the regions in HERV-K Gag corresponding to peptide sequences obtained by MS of 2-D separated proteins. Table 1 gives a summary of the peptide fragments obtained by MS analysis. The mass spectrometry did not allow for the identification of the exact cleavage site boundaries. Therefore, we once more purified

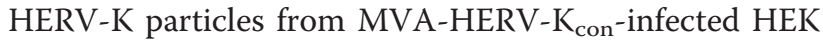
$293 \mathrm{~T}$ cells and separated the proteins by $2-\mathrm{D}$ electrophoresis and blotted them onto a PVDF membrane. Ponceau S-stained protein spots were cut out and Nterminal sequencing was performed. Table 2 gives a summary of the identified cleavage sites. The isoelectric point $(\mathrm{pI})$ and the molecular weight $(\mathrm{MW})$ were calculated for the protein fragments and correlated with the 2-D analysis (Figure 3A). Consequently, we propose a cleavage pattern of HERV-K as indicated in Figure 3B, with the following fragments:

- An apparently myristoylated MA of $15.2 \mathrm{kDa}$, which does not match the calculated pI of 9.1 but is rather

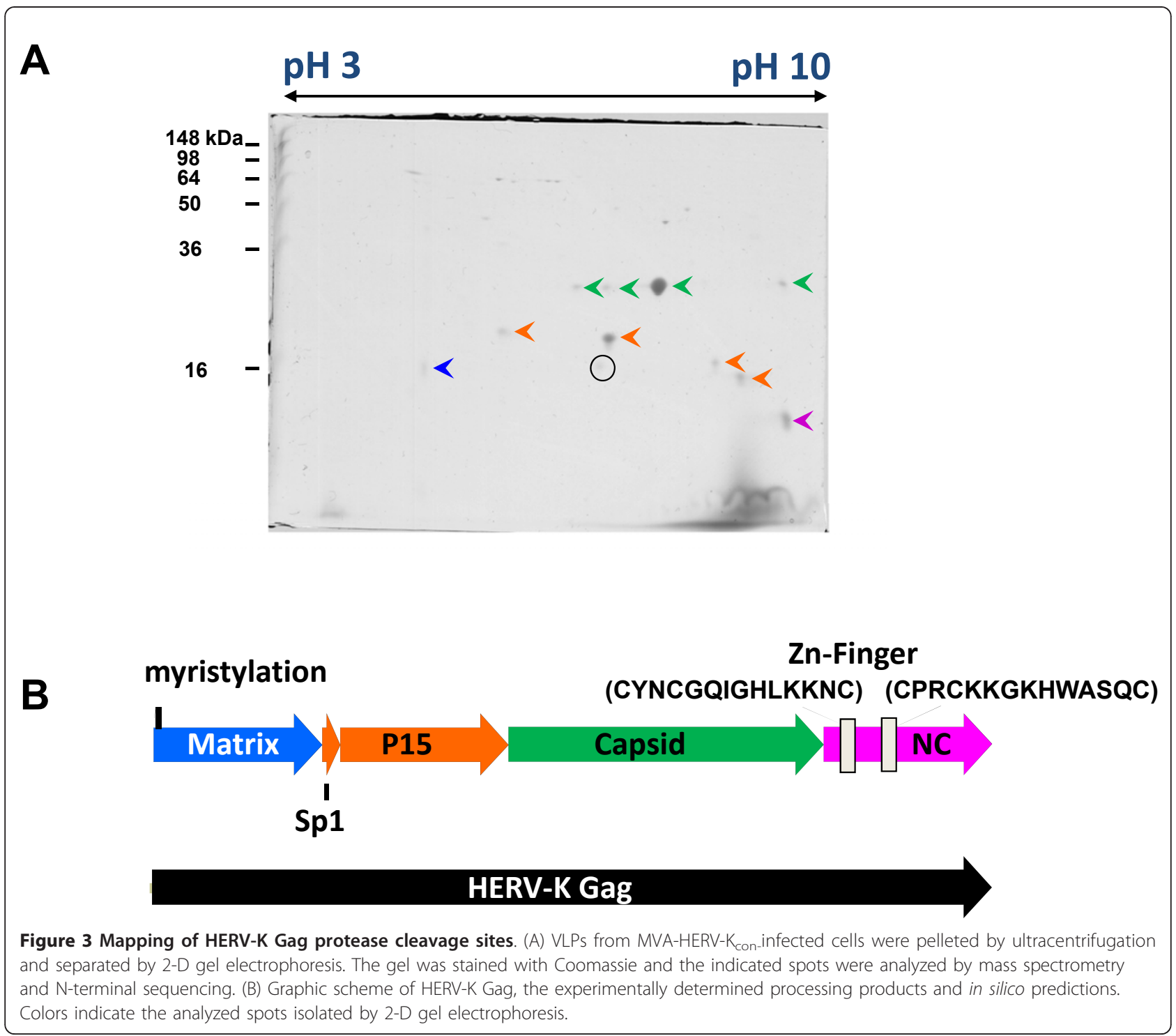


Table 1 Summary of the peptide fragments obtained by MS analysis

\begin{tabular}{|c|c|}
\hline $\begin{array}{c}\text { Gag } \\
\text { domains }\end{array}$ & $\begin{array}{l}\text { Peptides found by MS analysis } \\
\text { (AA position) }\end{array}$ \\
\hline Matrix & $\begin{array}{c}{ }_{31} \text { STKNLIKLFQIIEQFCPWFPEQGTLDLK }_{\mathbf{5 8}} \\
{ }_{74} \text { GNIIPLTTWNDWAIIKAALEPFQTEEDSVSVSDAPGSCIIDCNENTR }_{120}\end{array}$ \\
\hline P15 & $\begin{array}{c}{ }_{171} \text { VGPSESKPRGTSPLPAGQVPVTLQPQK }_{197} \\
{ }_{241} \text { APYPQPPTR }_{\mathbf{2 4 9}} \\
{ }_{260} \mathrm{QGSELHEIIDK}_{\mathbf{2 7 0}} \\
\end{array}$ \\
\hline Capsid & $\begin{array}{c}{ }_{324} \text { QYGPNSPYMRTLLDSIAHGHRLIPYDWEILAKSSLSPSQFLQFKTWWIDGVQEQVR } \\
\mathbf{3 7 9} \\
{ }_{383} \text { AANPPVNIDADQLLGIGQNWSTISQQALMQNEAIEQVR }_{\mathbf{4 2 0}} \\
{ }_{430} \text { IQDPGSTCPSFNTVRQGSKEPYPDFVAR }_{\mathbf{4 5 7}} \\
{ }_{499} \text { VPAGSDVISEYVK }_{\mathbf{5 1 1}}\end{array}$ \\
\hline$N C$ & $\begin{array}{l}{ }_{565} \text { QNITIQATTTGREPPDLCPR } \\
584 \\
{ }_{602} \text { NGQPLSGNEQR } 612\end{array}$ \\
\hline
\end{tabular}

The amino acid position within the Gag protein and an allocation to the Gag subfragments are indicated.

shifted to a low $\mathrm{pH}$ due to the myristoylation or phosphorylation.

- A short peptide SP1-HK.

- The p15, with a pI of 8.1 and MW of $15.1 \mathrm{kDa}$.

- The slightly over-presented, uncleaved product of SP1-HK and p15 with a $\mathrm{pI}$ of 5.9 and MW of 16.5 running in the middle of the 2-D gel (Figure 3A).

- CA, with a pI of 6.3 and MW $27.5 \mathrm{kDa}$. The identified $\mathrm{N}$-terminal cleavage site for $\mathrm{CA}$ matches the aromatic amino acid/proline sequence at the scissile bond consistently found at the $\mathrm{N}$-terminus of CA proteins of retroviruses.

- NC, with a pI of 9.6 and a MW of $14.6 \mathrm{kDa}$.

The $\mathrm{p} 15$ and the SP1-HK/p15 proteins display two spots in the 2-D analysis. This might result from protein phosphorylation, which changes the $\mathrm{pI}$ and can also affect protein mobility in SDS-PAGE analysis. In addition, we detected multiple spots of lower abundance for $\mathrm{CA}$ in the 2-D analysis, which might result from posttranslational modification, such as phosphorylation [14].

\section{Discussion}

The human genome encodes a large number of endogenous retroviral sequences, which encompass about $8 \%$ of the total genome. Human endogenous retroviruses (HERV) are transmitted vertically and are considered to be non-replicating, since almost all HERV genomes have inactivating mutations. Only HERV-K has retained its capability to form viral particles, but there are no signs of infectivity. Little is known about the biological function

Table 2 Summary of the sequences obtained from $\mathrm{N}$ terminal sequencing of HERV-K Gag cleavage products.

\begin{tabular}{clcc}
\hline Gag domains & \multicolumn{2}{c}{$\begin{array}{c}\text { Protease cleavage sites } \\
\text { (AA position) }\end{array}$} & Gag domains \\
\hline Matrix & LHCEY $_{134}$ & ${ }_{135}$ VAEPV & SP1-HK \\
SP1-HK & TQNVD $_{148}$ & ${ }_{149}$ YNQLQ & P15 \\
P15 & EAWQF $_{282}$ & ${ }_{283}$ PVTLE & Capsid \\
Capsid & QAITG $_{532}$ & ${ }_{533}$ WLGG & Nucleocapsid \\
\hline
\end{tabular}

of HERV-K expression, which has been detected in some tumor entities, such as human teratocarcinomas [4], and the basic virology has still not been investigated properly. In order to elucidate the hypothetical defect in Gag processing and-as a consequence-in virus infectivity, we mapped the previously unknown proteolytic cleavage sites of the HERV-K Gag polyprotein.

HERV-K virus-like particles based on the HERV-K consensus sequence were produced with the help of a recombinant poxvirus expressing the HERV-K Gag, the protease (PR) and the polymerase (Pol), and harvested from supernatants of the infected cells. The production of retroviruslike particles by vaccinia virus (VACV) expression of the gag/pol genes is well established and has been described previously, for example for HIV-1 [15], and transductioncompetent retroviral vectors have also been produced [16]. Moreover, VACV-produced HIV-1 particles have been used to test early HIV protease inhibitors and it could be shown that HIV-1-like particles released from inhibitor-treated cells contained almost exclusively the Gag precursor but no cleaved Gag products [17]. We used the highly attenuated MVA instead of replication-competent VACV which increased the safety of our work and provided proof of concept that MVA is also suitable for producing VLPs for biochemical analysis.

HERV-K particles were released from MVA-HERV$\mathrm{K}_{\text {con-infected cells, showing a typical gammaretrovirus }}$ morphology with assembly of the virus core at the cytoplasma membrane and extracellular particles of immature and mature morphology, with a condensed, acentric core (Figure 2C), similar to HERV-K113 particles produced by recombinant baculovirus expression [18] or HTDV/HERV-K expressed by teratocarcinoma cell lines [19]. HERV-K Gag proteolytic cleavage was dependent on the presence of its functional protease and potential poxvirus-encoded proteases could not cleave Gag in the absence of the viral protease (Figure 1).

In silico prediction of retroviral protease cleavage sites is not possible, because there is no clear consensus 
sequence motif. The substrate must be in an extended conformation to fit into the active site to be hydrolyzed [20]. Therefore, we identified the cleavage sites by a combination of 2-D electrophoresis, mass spectrometry and N-terminal sequencing, and could draw a map of Gag as illustrated in Figure 3B. The N-terminal cleavage site for HERV-K CA matched the sequence consistently found at the $\mathrm{N}$-terminus of all retroviral CA proteins. The other cleavage sites correspond well to the simplified version of a cleavage site as an amino acid stretch that is hydrophobic and both accessible and flexible, i.e. apparently the space between separately folded domains of Gag. HERV-K Gag is processed into MA, a short spacer peptide, p15, CA and NC. This structure is similar to mouse mammary tumor virus (MMTV). Interestingly, MMTV is morphologically a B-type virus where core assembly takes place inside the cytoplasma and only the preformed core migrates to the cell membrane. In addition, we found late (L)-domain motifs in the p15 protein of HERV-K, similar to Rous sarcoma virus, murine leukemia virus, and Mason-Pfizer monkey virus (MPMV), in which the L-domains also reside between $\mathrm{MA}$ and CA. In contrast, the late domains of HIV are located in the C-terminal p6 protein of Gag. L-domains are needed for efficient pinching off of the budding viral particles and different retroviruses utilize different viral proteins to accomplish late budding function [21].

\section{Conclusion}

The retroviral Gag protein is of high importance for retroviruses: it mediates the intracellular transport to the cell membrane, directs assembly of virus particles, and facilitates the budding of the viral particles. Blast alignment of all HERV-K Gag sequences showed that the cleavage sites identified here are highly conserved, and we could not detect a sequence change in the cleavage sites of any of the genomes available in the databases. This suggests that cleavable Gag proteins could be expressed by all HERV-K genomes and that the defect in replication must reside at a different step in the viral life cycle. On the other hand, this also suggests that recombination between HERV-K genomes or other retroviruses like HIV or XMRV [22] might give rise to novel infectious viral genomes able to reinfect human cells.

Note added in proof:

The same cleavage sites were obtained from Norbert Bannert's group (Robert Koch Institute, Berlin) using a different technical approach (George et al., manuscript in preparation).

\section{Methods}

\section{Cell culture}

HEK 293T, BHK21, RK-13 and NIH3T3 cells were cultured in complete Dulbecco's modified Eagle's medium
(DMEM) containing 10\% fetal bovine serum, penicillin (50 U/ml), streptomycin $(50 \mu \mathrm{g} / \mathrm{ml})$, and L-glutamine (2 $\mathrm{mM})$, and propagated by standard techniques. Transient MVA expressions were performed by transfection of the transfer plasmid and subsequent MVA infection at an MOI of 5. Transfections were performed with Fugene ${ }^{\mathrm{TM}}$ (Roche/Mannheim) in 6-well or $10 \mathrm{~cm}$ plates according to the manufacturer's instructions. At $24 \mathrm{~h}$ post transfection, cells were lysed for SDS-PAGE separation [23], fixed for EM or supernatants were collected for VLP purification. For collection of HERV-K $K_{\text {con }}$ VLPs, HEK $293 \mathrm{~T}$ cells were infected with MVA-HERV-K $\mathrm{K}_{\text {con }}$ at an MOI of 5 for $24 \mathrm{~h}$ under serum-free conditions before supernatants were harvested.

\section{Virus and plasmid construction}

The consensus HERV-K gag/pol gene HERV-Gag-PR-Pol [1] was kindly provided by Paul Bieniasz. Sequences were excised by Not 1 digestion, treated with T4-DNA polymerase to generate blunt ends, and cloned into the blunted BamH1 site of MVA expression plasmid pIII-pmH5 to generate the MVA vector plasmids pIII-HERV-K $\mathrm{K}_{\mathrm{con}}$. The protease-deficient clone pIII-HERV-K ${ }_{\text {con }}$ pro $^{-}$was generated via the following cloning steps: HERV- $\mathrm{K}_{\text {con }}$ sequences were excised from pCRVI/Gag-PR-Pol [1] by Not1 digestion and cloned into the Not 1 site of the pCMV-3 Flag plasmid (Agilent Technologies, Waldbronn). Subsequently, the unique Spe1 site located upstream of HERV$\mathrm{K} P R$ active center was cut, blunted and religated, resulting in a frameshift and introducing a stop codon. The HERV$\mathrm{K}_{\text {conpro }}{ }^{-}$sequence was cut out by Not1 and introduced into pIII-mH5 as described above to generate the MVA vector plasmid pIII-HERV-K conpro-. $^{-}$

The recombinant virus MVA-HERV-K $\mathrm{K}_{\text {con }}$ was generated in BHK21 cells by transfection with $1 \mu \mathrm{g}$ of plasmid DNA, infection at an MOI of 0.05 with MVA-IInew isolate, and plaque selection on RK-13 cells [24], [11]. The recombinant MVA genomes were analyzed by PCR to verify HERV-K gag/pol gene insertion and genetic stability. The production of HERV-K antigens was confirmed by Western blot analysis of various cell lysates harvested after infection with the recombinant MVA-HERV-K (data not shown). Multiple-step growth analysis in chicken embryo fibroblasts (CEFs) demonstrated that the replication capacities of MVA-HERV-K $K_{\text {con }}$ were comparable to non-recombinant MVA (data not shown).

\section{Ultracentrifugation of cell supernatants}

HERV-K VLPs were separated from MVA by filtration through $0.2 \mu \mathrm{m}$ filters and concentrated by ultracentrifugation of cell supernatants through a $30 \%$ sucrose cushion for $2 \mathrm{~h}$ at $4^{\circ} \mathrm{C}$ and $130,000 \times \mathrm{g}$. The viral pellet was resuspended in $150 \mu$ l rehydration buffer for 2-D analysis or in $50 \mu \mathrm{l}$ sample buffer for SDS-PAGE. 


\section{Two-dimensional electrophoresis}

HERV-K VLP-containing pellets obtained by ultracentri-

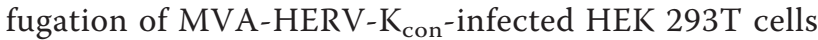
were resuspended in $150 \mu$ l rehydration buffer ( $8 \mathrm{M}$ urea; 1\% CHAPS; 10 mM DTT; 0.25\% ampholyte) and used to rehydrate a ReadyStrip IPG Strip pH 3-10 (Bio-Rad, Munich). Samples were isoelectrically focused using a standardized program (20 min $250 \mathrm{~V} ; 2$ h $4000 \mathrm{~V} ; 10000$ Vh $4000 \mathrm{~V}$ ). After isoelectric focusing, strips were equilibrated for the second dimension in equilibration buffer (6 M urea; 30\% glycerin; 2\% SDS; 50 mM Tris pH 8.8) plus $1 \%$ DTT (w/v) for $15 \mathrm{~min}$ and in equilibration buffer plus $20 \%$ iodoacetamide $(\mathrm{w} / \mathrm{v})$ for further $15 \mathrm{~min}$. Isoelectrically focused samples were separated via 15\% SDSPAGE with standard techniques followed by Western blot or Coomassie staining for mass spectrometry.

\section{Western blot}

Western blot was performed with a BIO-Rad semi-dry blotter. Proteins separated by SDS-PAGE were blotted onto PVDF membranes with $50 \mathrm{mM}$ sodium borate $\mathrm{pH}$ 9.0, 20\% methanol, and $0.1 \%$ SDS at $100 \mathrm{~mA}$ per membrane for $75 \mathrm{~min}$. Afterwards, membranes were either: (1) washed with water and stained with Ponceau S (Sigma Aldrich, Hamburg), and protein spots were cut out and washed extensively with water for $\mathrm{N}$-terminal Edman amino acid sequencing http://proteome-factory.com; or (2) blocked with Roti-Block ${ }^{\mathrm{TM}}$ and proteins were detected with $\alpha$-HERV-K capsid monoclonal antibodies [9] and the ECL detection system (Amersham, Freiburg).

\section{Electron microscopy}

Cells were fixed with $2.5 \%$ glutaraldehyde in culture medium for $45 \mathrm{~min}$ at room temperature. After washing in PBS, cells were scraped off the culture disk and gently mixed with $2 \%$ warm liquid agarose. After cooling and gelling, small agarose blocks containing the cells were cut. These blocks were post-fixed with $2 \%$ osmium tetroxide in PBS and treated with $1 \%$ tannic acid to improve visibility of viral surface proteins [25]. Cells were then dehydrated in a graded series of ethanol and finally embedded in epoxy resin (Sigma-Aldrich, Steinheim) according to standard preparation protocols. Ultrathin sections were stained with $2 \%$ uranylacetate for $15 \mathrm{~min}$ followed by $2 \%$ lead citrate for $5 \mathrm{~min}$.

\section{Mass spectrometry}

Protein spots of interest were excised from Coomassiestained 2-D gels, destained, reduced, alkylated and digested with trypsin as described elsewhere [26]. As a modification of this method, peptides were eluted with $25 \mathrm{mM} \mathrm{NH}_{4} \mathrm{HCO}_{3}$ in $10 \%$ acetonitrile $(\mathrm{ACN})$ and the digestion was stopped by adding $5 \%$ formic acid. The peptides were separated with a nano-Acquity UPLC
(Waters, Milford, USA) using a $5 \mu \mathrm{m}$ symmetry $180 \mu \mathrm{m}$ $\times 20 \mathrm{~mm}$ c18 pre-column and a $1.7 \mu \mathrm{m}$ BEH130 100 $\mu \mathrm{m} \times 100 \mathrm{~mm}$ c18 separation column at a flow rate of $500 \mathrm{nl}$ applying a gradient of $30 \mathrm{~min}(3 \% \mathrm{ACN}$ to $40 \%$ ACN). The UPLC was coupled to a Nano-ESI Synapt mass spectrometer (Waters) operated in $\mathrm{V}$ mode, acquiring $\mathrm{MS}^{\mathrm{E}}$ data and applying standard parameters. Data analysis was performed with the Protein Lynx Global Server Version 2.3 (Waters). Protein hits were accepted at a false positive rate of less than $4 \%$; peptide mass accuracy was 5 ppm or better.

\section{Acknowledgements}

This work was supported by a grant from the Deutsche

Forschungsgemeinschaft to the graduate school GRK 1172. We thank Heike Strobel and Regina Eberle for excellent technical assistance, Gerd Sutter for providing MVA, Paul Bieniasz for the pCRVI/Gag-PR-Pol plasmid and Roswitha and Johannes Löwer for helpful discussions.

\section{Authors' contributions}

BK carried out the studies and helped to draft the manuscript; KB performed the EM studies, and AR did the mass spectrometry. BS conceived, designed and coordinated the study and drafted the manuscript. All authors read and approved the final manuscript.

\section{Competing interests}

The authors declare that they have no competing interests.

Received: 14 December 2010 Accepted: 23 March 2011

Published: 23 March 2011

\section{References}

1. Lee YN, Bieniasz PD: Reconstitution of an infectious human endogenous retrovirus. PLOS Pathog 2007, 3:e10

2. Bannert N, Kurth R: The evolutionary dynamics of human endogenous retroviral families. Annu Rev Genomics Hum Genet 2006, 7:149-173.

3. Magin C, Lower R, Lower J: cORF and RcRE, the Rev/Rex and RRE/RxRE homologues of the human endogenous retrovirus family HTDV/HERV-K. J Virol 1999, 73:9496-9507.

4. Lower R, Lower J, Frank H, Harzmann R, Kurth R: Human teratocarcinomas cultured in vitro produce unique retrovirus-like viruses. J Gen Virol 1984, 65(Pt 5):887-898.

5. Buscher K, Trefzer U, Hofmann M, Sterry W, Kurth R, Denner J: Expression of human endogenous retrovirus $\mathrm{K}$ in melanomas and melanoma cell lines. Cancer Res 2005, 65:4172-4180.

6. Wang-Johanning F, Liu J, Rycaj K, Huang M, Tsai K, Rosen DG, Chen DT, Lu DW, Barnhart KF, Johanning GL: Expression of multiple human endogenous retrovirus surface envelope proteins in ovarian cancer. Int J Cancer 2007, 120:81-90.

7. Herbst H, Kuhler-Obbarius C, Lauke H, Sauter M, Mueller-Lantzsch N, Harms D, Loning T: Human endogenous retrovirus (HERV)-K transcripts in gonadoblastomas and gonadoblastoma-derived germ cell tumours. Virchows Arch 1999, 434:11-15.

8. Dewannieux M, Harper F, Richaud A, Letzelter C, Ribet D, Pierron G, Heidmann T: Identification of an infectious progenitor for the multiplecopy HERV-K human endogenous retroelements. Genome Res 2006, 16:1548-1556.

9. Bieda K, Hoffmann A, Boller K: Phenotypic heterogeneity of human endogenous retrovirus particles produced by teratocarcinoma cell lines. J Gen Virol 2001, 82:591-596.

10. Mueller-Lantzsch N, Sauter M, Weiskircher A, Kramer K, Best B, Buck M Grasser F: Human endogenous retroviral element K10 (HERV-K10) encodes a full-length gag homologous $73-\mathrm{kDa}$ protein and a functional protease. AIDS Res Hum Retroviruses 1993, 9:343-350.

11. Staib C, Drexler I, Sutter G: Construction and isolation of recombinant MVA. Methods Mol Biol 2004, 269:77-100. 
12. Lee SK, Nagashima K, Hu WS: Cooperative effect of gag proteins p12 and capsid during early events of murine leukemia virus replication. J Virol 2005, 79:4159-4169.

13. Schommer S, Sauter M, Krausslich HG, Best B, Mueller-Lantzsch N: Characterization of the human endogenous retrovirus $\mathrm{K}$ proteinase. J Gen Virol 1996, 77(Pt 2):375-379.

14. Gottwein $\mathrm{E}$, Krausslich HG: Analysis of human immunodeficiency virus type 1 Gag ubiquitination. J Virol 2005, 79:9134-9144.

15. Karacostas V, Nagashima K, Gonda MA, Moss B: Human immunodeficiency virus-like particles produced by a vaccinia virus expression vector. Proc Natl Acad Sci USA 1989, 86:8964-8967.

16. Konetschny C, Holzer GW, Urban C, Hammerle T, Mayrhofer J, Falkner FG: Generation of transduction-competent retroviral vectors by infection with a single hybrid vaccinia virus. J Virol 2003, 77:7017-7025.

17. McQuade TJ, Tomasselli AG, Liu L, Karacostas V, Moss B, Sawyer TK, Heinrikson RL, Tarpley WG: A synthetic HIV-1 protease inhibitor with antiviral activity arrests HIV-like particle maturation. Science 1990, 247:454-456.

18. Tonjes RR, Boller K, Limbach C, Lugert R, Kurth R: Characterization of human endogenous retrovirus type $\mathrm{K}$ virus-like particles generated from recombinant baculoviruses. Virology 1997, 233:280-291.

19. Lower R, Lower J, Kurth R: The viruses in all of us: characteristics and biological significance of human endogenous retrovirus sequences. Proc Natl Acad Sci USA 1996, 93:5177-5184.

20. Vogt VM: Proteolytic processing and particle maturation. Curr Top Microbiol Immunol 1996, 14:95-131.

21. Martin-Serrano J: The role of ubiquitin in retroviral egress. Traffic 2007, 8:1297-1303.

22. Enserink M: Chronic fatigue syndrome. New XMRV paper looks good, skeptics admit-yet doubts linger. Science 2010, 329:1000.

23. Schnierle BS, Moritz D, Jeschke M, Groner B: Expression of chimeric envelope proteins in helper cell lines and integration into Moloney murine leukemia virus particles. Gene Ther 1996, 3:334-42.

24. Staib C, Lowel M, Erfle V, Sutter G: Improved host range selection for recombinant modified vaccinia virus Ankara. Biotechniques 2003, 34:694-6.

25. Gelderblom HR, Hausmann EH, Ozel M, Pauli G, Koch MA: Fine structure of human immunodeficiency virus (HIV) and immunolocalization of structural proteins. Virology 1987, 156:171-176.

26. Albrecht M, Alessandri S, Conti A, Reuter A, Lauer I, Vieths S, Reese G: High level expression, purification and physico- and immunochemical characterisation of recombinant Pen a 1: a major allergen of shrimp. Mol Nutr Food Res 2008, 52(Suppl 2):S186-S195.

doi:10.1186/1742-4690-8-21

Cite this article as: Kraus et al: Characterization of the human endogenous retrovirus $\mathrm{K}$ Gag protein: identification of protease cleavage sites. Retrovirology 2011 8:21.

\section{Submit your next manuscript to BioMed Central and take full advantage of:}

- Convenient online submission

- Thorough peer review

- No space constraints or color figure charges

- Immediate publication on acceptance

- Inclusion in PubMed, CAS, Scopus and Google Scholar

- Research which is freely available for redistribution

Submit your manuscript at www.biomedcentral.com/submit
Biomed Central 